\title{
A no-go theorem for slowly rotating black holes in Hořava-Lifshitz gravity
}

\author{
Enrico Barausse ${ }^{1}$ and Thomas P. Sotiriou ${ }^{2}$ \\ ${ }^{1}$ Department of Physics, University of Guelph, Guelph, Ontario, N1G 2W1, Canada \\ ${ }^{2}$ SISSA, Via Bonomea 265, 34136, Trieste, Italy and INFN, Sezione di Trieste, Italy
}

(Dated: April 1, 2022)

\begin{abstract}
We consider slowly rotating, stationary, axisymmetric black holes in the infrared limit of HořavaLifshitz gravity. We show that such solutions do not exist, provided that they are regular everywhere apart from the central singularity. This has profound implications for the viability of the theory, considering the astrophysical evidence for the existence of black holes with non-zero spin.
\end{abstract}

Black-hole $(\mathrm{BH})$ spacetimes have event horizons that act as causal boundaries. This property is intimately related to the causal structure of relativistic gravity theories, such as general relativity (GR). It is, therefore, reasonable to ask whether BHs can actually exist in theories that exhibit violations of Lorentz symmetry.

Interest in Lorentz-violating (LV) gravity theories stems from the fact that constraints on Lorentz violations in gravity are significantly weaker than in the matter sector, since the gravity sector is weakly coupled. In fact, Einstein-aether theory (æ-theory), which is GR coupled to a unit timelike vector field, was proposed as a benchmark for quantifying Lorentz violations in gravity [1. More recently, a more direct motivation for LV gravity came from Ref. [2, where a framework for constructing a power-counting renormalizable gravity theory was presented. The desirable ultraviolet (UV) behavior is achieved by including higher-order spatial derivatives in the gravitational action, but only second-order time derivatives in order to avoid loss of unitarity. Terms with at least $2 d$ spatial derivatives, where $d$ is the number of spatial dimensions, are required in order to achieve power-counting renormalizability [2 4].

The corresponding theory is known as Hořava-Lifshitz (HL) gravity (see e.g. [5] for a brief review). The action is straightforwardly constructed once a preferred foliation is imposed, and is invariant under the reduced set of diffeomorphisms that leave this foliation intact, $T \rightarrow \tilde{T}(T)$ and $x^{i} \rightarrow \tilde{x}^{i}\left(T, x^{i}\right)$. In $3+1$ dimensions one then has [6]

$$
S_{H L}=\frac{M_{\mathrm{Pl}}}{2} \int d T d^{3} x N \sqrt{h}\left(L_{2}+\frac{1}{M_{\star}^{2}} L_{4}+\frac{1}{M_{\star}^{4}} L_{6}\right),
$$

where $M_{\mathrm{Pl}}$ is the Planck scale, $h$ is the determinant of the metric $h_{i j}$ induced on the spacelike hypersurfaces and

$$
L_{2}=K_{i j} K^{i j}-\lambda K^{2}+\xi^{(3)} R+\eta a_{i} a^{i},
$$

where $K$ is the trace of the extrinsic curvature $K_{i j}$, ${ }^{(3)} R$ is the Ricci scalar of $h_{i j}, N$ is the lapse function, $a_{i}=\partial_{i} \ln N$, and $\lambda, \xi$ and $\eta$ are dimensionless parameters. $L_{4}$ and $L_{6}$ denote respectively collections of all the 4th-order and 6th-order operators, while $M_{\star}$ is the scale that suppresses these operators. $L_{4}$ and $L_{6}$ contain a very large number $\left(\sim 10^{2}\right)$ of operators and independent coupling parameters and this can be considered as an unappealing feature of the theory. However, here we will focus on the infrared (IR) limit of the theory, which depends only on $L_{2}$. The only feature associated with $L_{4}$ and $L_{6}$ that will concern us is the fact that dispersion relations cease to be linear at energies around $M_{\star}$. We will also not consider versions of the theory where the action is required to satisfy extra restrictions and symmetries, see e.g. Refs. 2, 17-10 and Ref. [5 for a brief review.

The deviations of $L_{2}$ from GR are measured by $|1-\lambda|$, $|1-\xi|$ and $\eta$. The limit to GR is not smooth, as HL gravity presents an extra scalar mode due to the reduced symmetry with respect to GR. This scalar mode can exhibit instabilities, have negative energy or get strongly coupled at unacceptably low energies [11 14. However, in a significant part of the parameter space the theory is free from pathologies and viable in the IR [15]. Avoiding strong coupling imposes an upper bound on $M_{\star}$ [13, 16] (see Refs. [13, 17] for the implications of this bound).

If one chooses to restore diffeomorphism invariance, the scalar mode manifests as a foliation-defining scalar field and the IR limit of HL gravity can take the form of æ-theory with the extra condition that the aether be hypersurface orthogonal at the level of the action [18] (the "covariantization" can be extended beyond the IR limit [19]). The corresponding action is

$$
S_{æ}=\frac{M_{æ}}{2} \int d^{4} x \sqrt{-g}\left(-R-M_{\mu \nu}^{\alpha \beta} \nabla_{\alpha} u^{\mu} \nabla_{\beta} u^{\nu}\right),
$$

where $g$ is the determinant of the metric $g_{\mu \nu}, \nabla_{\mu}$ is the associated covariant derivative, $R$ is the Ricci scalar of this metric,

$$
M_{\mu \nu}^{\alpha \beta} \equiv c_{1} g^{\alpha \beta} g_{\mu \nu}+c_{2} \delta_{\mu}^{\alpha} \delta_{\nu}^{\beta}+c_{3} \delta_{\nu}^{\alpha} \delta_{\mu}^{\beta}+c_{4} u^{\alpha} u^{\beta} g_{\mu \nu},
$$

$c_{1}$ to $c_{4}$ are dimensionless parameters, and locally the aether is given in terms of the foliation-defining scalar $T$ as

$$
u_{\mu}=\frac{\partial_{\mu} T}{\sqrt{g^{\alpha \beta} \partial_{\alpha} T \partial_{\beta} T}} .
$$

The correspondence of parameters with action (1) is

$$
\frac{M_{\circledast 2}}{M_{\mathrm{pl}}}=\xi=\frac{1}{1-c_{13}}, \quad \lambda=\frac{1+c_{2}}{1-c_{13}}, \quad \eta=\frac{c_{14}}{1-c_{13}},
$$


where $c_{i j}=c_{i}+c_{j} . c_{4}$ (or $c_{1}$ or $c_{3}$ ) may be set to zero without loss of generality as long as eq. (5) holds. However, we have not done so in the equations above, in order to have a direct comparison with general æ-theory, in which eq. (5) does not hold, but instead the aether is a full-fledged vector that satisfies the constraint $u^{\mu} u_{\mu}=1$.

In Ref. [20] it has been shown that æ-theory admits a one-parameter family of asymptotically flat, static, spherically symmetric solutions, which are regular everywhere apart from the central singularity and have a metric horizon (see also Ref. [21] for earlier work). Since spherically symmetric vector fields are always hypersurface orthogonal, these are also solutions of the IR limit of HL gravity.

These solutions present a metric horizon, which acts as a causal boundary for matter fields coupled minimally to the metric, and additional horizons, which act as causal boundaries for the gravity-sector modes. Thus, from an IR perspective these are indeed BHs. However, in HL gravity, dispersion relations cease to be linear at the scale $M_{\star}$, so short-wavelength perturbations travel at arbitrarily high speeds in the preferred frame and penetrate all horizons. One expects the same to happen in any sensible UV completion of æ-theory. Because $M_{\star}$ corresponds to a length scale much smaller than the horizon of astrophysical BHs, one does not expect significant corrections to the solutions of Ref. 20 near the horizons. Nevertheless, from a conceptual viewpoint, even though these solutions should be very close to those of the full UVcomplete theory, the interpretation of the various horizons as true causal boundaries would be incorrect. As a result, the very concept of a $\mathrm{BH}$ would not survive.

A striking feature of the solutions of Ref. [20] is the existence of a hypersurface that is orthogonal to the aether, and which lies inside the various horizons and therefore cloaks the singularity. This hypersurface can be interpreted as a constant-time hypersurface as measured in the preferred frame defined by the aether (or as a constant $T$ hypersurface in HL gravity), and is also a constant $r$ hypersurface, where $r$ is the Schwarzschild coordinate. This hypersurface acts as a universal horizon, as it constitutes a causal boundary for all modes, irrespectively of their propagation speed [20]. The existence of this hypersurface has been shown also in Ref. 22] in the decoupling limit. However, it has also been shown there that this universal horizon appears to be non-linearly unstable against non-spherically symmetric perturbations in the infrared limit of HL gravity (although not in $æ-$ theory). Thus, the question that we want to address is: do BHs really exist in HL gravity, if one abandons the idealized assumption of spherical symmetry? In this Letter we will focus on slowly rotating BHs, which in GR can be obtained from the Schwarzschild solution by expanding the field equations in the rotation parameter 23 .

We first show that the static, spherically symmetric, asymptotically flat solutions for $æ$-theory exactly coin- cide with those of HL gravity, assuming that the aether $u^{\mu}$ in $æ$-theory, and the scalar $T$ in HL gravity asymptote to their trivial configuration in Minkowski space, i.e. $u^{\mu}=\delta_{t}^{\mu}$ and $T=t$ in the preferred frame (see also Ref. [15). Therefore, the solutions found in Ref. 20 are the full set of static, spherical and asymptotically flat BHs of HL gravity. This is not obvious because the equivalence between æ-theory and the infrared limit of HL gravity requires that the aether be hypersurfaceorthogonal at the level of the action, and as a result the two theories do not have the same field equations. In fact, variation of (3) with respect to $u^{\mu}$ without assuming eq. (5) gives a set of four equations $\mathbb{E}_{\mu}=0$, whereas variation of (3) with respect to $T$ yields

$$
\partial_{\mu}\left(\frac{1}{\sqrt{\nabla^{\alpha} T \nabla_{\alpha} T}} \sqrt{-g} \boxplus^{\mu}\right)=0 .
$$

Solutions of $\mathbb{E}^{\mu}=0$ satisfy also eq. (7), so hypersurface orthogonal solutions of æ-theory will also be solutions of HL gravity. To show the converse, let us first note that once we impose spherical symmetry and staticity, $\mathbb{E}^{\theta}=$ $\mathbb{E}^{\varphi}=0$ identically, and $\mathbb{E}^{r}=0$ implies $\mathbb{E}^{t}=0$. Thus, it suffices to prove that eq. (7) yields $\mathbb{E}^{r}=0$. Eq. (7) now involves only $r$ and $\theta$ derivatives, so integrating between $r=r_{1}$ and $r=r_{2}$ and using the divergence theorem yields

$$
\left.\int_{\theta=0}^{\theta=\pi} \frac{1}{\sqrt{\nabla^{\alpha} T \nabla_{\alpha} T}} \sqrt{-g} \mathbb{E}^{r} d \theta\right|_{r=r_{1}} ^{r=r_{2}}=0
$$

Sending now $r_{2} \rightarrow \infty$, from asymptotic flatness we get $\sqrt{\nabla^{\alpha} T \nabla_{\alpha} T} \sim 1, \sqrt{-g} \sim r^{2} \cos \theta$ and $\mathbb{E}^{r} \sim \partial^{2} u \sim 1 / r^{3}$, hence $\sqrt{-g} \mathbb{E}^{r} / \sqrt{\nabla^{\alpha} T \nabla_{\alpha} T} \sim 1 / r \rightarrow 0$. Therefore, at $r=r_{1}$ we must have $\mathbb{E}^{r}=0$.

We now turn our attention to rotating BHs. The most general slowly rotating, stationary, axisymmetric metric can be written, in a suitable coordinate system, as 23 .

$$
\begin{aligned}
d s^{2}= & f(r) d t^{2}-\frac{B(r)^{2}}{f(r)} d r^{2}-r^{2}\left(d \theta^{2}+\sin ^{2} \theta d \varphi^{2}\right) \\
& +\epsilon r^{2} \sin ^{2} \theta \Omega(r, \theta) d t d \varphi+\mathcal{O}\left(\epsilon^{2}\right),
\end{aligned}
$$

where $\epsilon$ is the book-keeping parameter of the expansion. Since we are interested in slowly rotating BHs in HL gravity, $f(r)$ and $B(r)$ are given by the solutions of Ref. [20].

As for $T$, given that it appears in the action only through $u_{\mu}$, one cannot exclude a dependence on $t$ or $\varphi$, provided that $\partial_{t} u_{\mu}=\partial_{\varphi} u_{\mu}=0$. In fact, given that $u^{\mu}$ is timelike a $t$-dependence is necessary. Hypersurface orthogonality for $u^{\mu}$ implies vanishing vorticity, and thus yields $u_{\varphi}=\ell u_{t}$, where $\ell=$ constant can be interpreted as the angular momentum of the aether per unit energy, as seen at infinity. Clearly, $\ell$ can be made to vanish if we make our coordinate system corotate with the aether at infinity, i.e. if we perform the coordinate change $t^{\prime}=t-\ell \varphi$, we have $u_{\varphi}=0$. Provided that 
$\ell=\mathcal{O}(\epsilon)$ (which follows from the slow-rotation assumption), it is easy to see that such a coordinate change leaves the ansatz (9) invariant modulo a redefinition of $\Omega(r, \theta)$. Without loss of generality we then set $u_{\varphi}=0$ and write

$$
\boldsymbol{u}=\frac{1+f A^{2}}{2 A} d t+\frac{B}{2 A}\left(\frac{1}{f}-A^{2}\right) d r+\mathcal{O}(\epsilon)^{2},
$$

where we have also used $u_{\theta}=\mathcal{O}\left(\epsilon^{2}\right)$ and $g^{\mu \nu} u_{\mu} u_{\nu}=$ 1. $A$ is the aether component $u^{v}$ in ingoing EddingtonFinkelstein coordinates, used in Ref. 20], and we have suppressed the $r$-dependence of $A, B$ and $f$ to lighten the notation. Note, however, that in general $u^{\varphi} \neq 0$.

For $T$, the above translates into $T=t+\tau(r, \theta)$, if one also uses invariance under $T \rightarrow \tilde{T}(T)$. Assuming $u_{\varphi} \neq 0$ would also imply a linear dependence of $T$ on $\varphi$, and $T$ would not be single-valued. This would jeopardize the equivalence between hypersurface orthogonal æ-theory and HL gravity, which requires that $T$ be identified with the time coordinate in the preferred foliation.

The action (3) remains unchanged under the set of field redefinitions $g_{\alpha \beta}^{\prime}=g_{\alpha \beta}+\left(s^{2}-1\right) u_{\alpha} u_{\beta}, u^{\prime \alpha}=s^{-1} u^{\alpha}$, if the $c_{i}$ are replaced with new couplings $\tilde{c}_{i}\left(c_{i}\right)$ [24]. $g_{\alpha \beta}^{\prime}$ and $u_{\mu}^{\prime}$ are still described by the ansätze (9) and (10) (after a suitable coordinate transformation). For $s=s_{0}, s_{0}$ being the speed of the spin- 0 mode, $g_{\alpha \beta}^{\prime}$ becomes the effective metric on which spin-0 excitations propagate 21. Hence, the horizon of $g_{\alpha \beta}^{\prime}$ coincides with the causal boundary of the solutions of Ref. 20] for the spin-0 mode. We find it convenient to work with these redefined fields, as in Refs. [20, 21]. After the field redefinitions, one can still exploit hypersurface orthogonality for $u^{\mu}$ and set $\tilde{c}_{4}=0$.

As in Ref. [20, we restrict ourselves to the part for the (physical) parameter space for which: (i) all propagating modes are stable and have positive energy, (ii) vacuum Cherenkov radiation by matter is avoided [25], and (iii) there is complete agreement with GR at the first post-Newtonian order (vanishing preferred-frame parameters) [15. The last condition requires that $\eta=2(\xi-1)$ and restricts the parameter space to two dimensions, see Fig. 1. The corresponding static, spherically symmetric BHs, which act as "seeds" for the slowly rotating BHs studied here, are presented in section VB of Ref. [20].

At first order in $\epsilon$, there are three non-trivial independent HL field equations:

$$
\begin{aligned}
& \frac{\tilde{c}_{13}}{8 r^{3} A^{3} B f^{2}}\left\{f\left[2 \partial_{\theta} \Omega(r, \theta)\left(A-r A^{\prime}\right)+r A \partial_{r} \partial_{\theta} \Omega(r, \theta)\right]\right. \\
& -f^{3} A^{4}\left[2\left(r A^{\prime}+A\right) \partial_{\theta} \Omega(r, \theta)+r A \partial_{r} \partial_{\theta} \Omega(r, \theta)\right] \\
& \left.-r A f^{\prime} \partial_{\theta} \Omega(r, \theta)\left(1+A^{4} f^{2}\right)\right\}=0 \\
& \frac{1}{r^{2} f}\left\{\frac{1}{2}\left[-\partial_{\theta}^{2} \Omega(r, \theta)-3 \cot \theta \partial_{\theta} \Omega(r, \theta)\right]+k_{0} \Omega(r, \theta)\right. \\
& \left.\quad+k_{1} \partial_{r} \Omega(r, \theta)+k_{2} \partial_{r}^{2} \Omega(r, \theta)\right\}=0 \\
& q_{0} \Omega(r, \theta)+q_{1} \partial_{r} \Omega(r, \theta)+q_{2} \partial_{r}^{2} \Omega(r, \theta)=0
\end{aligned}
$$

where a prime denotes differentiation with respect to the argument. Also, $k_{i}, q_{i}, i=0,1,2$ are functions of $\tilde{c}_{1}$ and $\tilde{c}_{3}$, as well as of $A, f$ and $B$ and their derivatives.

Now, $\Omega(r, \theta)=\Omega_{0}=$ constant must be a solution to these equations because it is just the spherically symmetric static metric, transformed under the coordinate change $\varphi^{\prime}=\varphi+\Omega_{0} t$. Thus, $k_{0}=q_{0}=0$ when one uses the spherically symmetric static solution.

In the GR limit $\tilde{c}_{1}=\tilde{c}_{3}=0$, one has $q_{1}=q_{2}=$ 0 , as well as $k_{1}=r f\left(r B^{\prime}-4 B\right) /\left(2 B^{3}\right)$ and $k_{2}=$ $-r^{2} f /\left(2 B^{2}\right)$. Using the Schwarzschild solution $B=1$ and $f=1-2 M / r$, eq. 12 then gives

$$
\begin{aligned}
-(r-2 M)\left[4 \partial_{r} \Omega(r, \theta)+r \partial_{r}^{2} \Omega(r, \theta)\right] \\
=\partial_{\theta}^{2} \Omega(r, \theta)+3 \cot \theta \partial_{\theta} \Omega(r, \theta) .
\end{aligned}
$$

Solving by separation of variables and imposing regularity at the poles, one finds the expected solutions $\Omega(r, \theta)=$ constant and $\Omega(r, \theta)=\Omega_{\mathrm{H}}(2 M / r)^{3}\left(\Omega_{\mathrm{H}}=\right.$ constant being the horizon's angular velocity), which is the slowly rotating limit of the Kerr solution.

In HL gravity instead there are extra equations, without any extra function to be determined. This is crucial as taking a linear combinations of eqs. $120-13$ can be used in order to eliminate $\partial_{r}^{2} \Omega(r, \theta)$ and obtain

$$
\begin{aligned}
& \frac{\tilde{c}_{13}\left(A^{4} f^{2}-1\right)}{16 A^{2} B^{3}}\left\{\partial_{\theta}^{2} \Omega(r, \theta)+3 \cot \theta \partial_{\theta} \Omega(r, \theta)\right. \\
& \left.+2 \tilde{c}_{1} r^{2}\left(\tilde{c}_{13}-1\right) f \frac{\left(A^{2} f-1\right) A^{\prime}+A^{3} f^{\prime}}{A B^{2} \tilde{c}_{13}\left(A^{2} f+1\right)} \partial_{r} \Omega(r, \theta)\right\}=0 .
\end{aligned}
$$

Solving naively by separation of variables and imposing regularity at the poles, one finds $\Omega=$ constant (i.e. the spherical solution in rotating coordinates) as the only solution. We now show that this is indeed the only solution to eq. 15), even if one does not assume $\Omega(r, \theta)=R(r) Q(\theta)$. At the horizon $r_{\mathrm{H}}, f\left(r_{\mathrm{H}}\right)=0$ but $f^{\prime}\left(r_{\mathrm{H}}\right) \neq 0$ and $A\left(r_{\mathrm{H}}\right) \neq 0$ [20], so eq. 111] yields $\partial_{\theta} \Omega\left(r_{\mathrm{H}}, \theta\right)=0$, hence $\Omega\left(r_{\mathrm{H}}, \theta\right)=\omega_{0}=$ constant. Because the spherical solution is regular at the horizon [20, assuming analyticity, we can write

$$
\begin{gathered}
f(r)=\sum_{n=1}^{\infty} \frac{f^{(n)}\left(r_{\mathrm{H}}\right)}{n !}\left(r-r_{\mathrm{H}}\right)^{n}, \\
B(r)=B\left(r_{\mathrm{H}}\right)+\sum_{n=1}^{\infty} \frac{B^{(n)}\left(r_{\mathrm{H}}\right)}{n !}\left(r-r_{\mathrm{H}}\right)^{n}, \\
A(r)=A\left(r_{\mathrm{H}}\right)+\sum_{n=1}^{\infty} \frac{A^{(n)}\left(r_{\mathrm{H}}\right)}{n !}\left(r-r_{\mathrm{H}}\right)^{n},
\end{gathered}
$$

and assuming that the slowly-rotating solution is also regular and analytic, we can write

$$
\Omega(r, \theta)=\omega_{0}+\sum_{n=1}^{\infty} \omega_{n}(\cos \theta)\left(r-r_{\mathrm{H}}\right)^{n}
$$




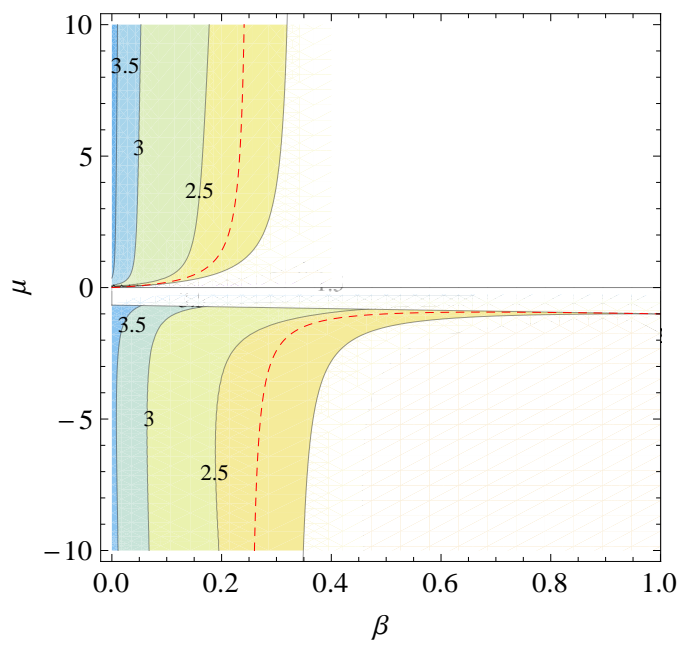

FIG. 1. Graphic representation of the viable part of the parameter space, where $\beta=(\xi-1) / \xi$ and $\mu=(\lambda-\xi) / \xi$. Contours represent the value of $S$. The dashed line represents $\tilde{c}_{13}=0$.

(Note that analyticity is also used to prove the uniqueness of the Kerr-Newman BH solution in GR 26].) At the lowest order in $r-r_{\mathrm{H}}$, eq. 15) gives

$$
\frac{\tilde{c}_{13}\left[-4 \cos \theta \omega_{1}^{\prime}(\cos \theta)+\sin ^{2} \theta \omega_{1}^{\prime \prime}(\cos \theta)\right]}{2 \tilde{c}_{1}\left(1-\tilde{c}_{13}\right)}=S \omega_{1}(\cos \theta),
$$

where

$$
S \equiv \frac{r_{\mathrm{H}}^{2} f^{\prime}\left(r_{\mathrm{H}}\right)\left[A\left(r_{\mathrm{H}}\right)^{3} f^{\prime}\left(r_{\mathrm{H}}\right)-A^{\prime}\left(r_{\mathrm{H}}\right)\right]}{A\left(r_{\mathrm{H}}\right) B\left(r_{\mathrm{H}}\right)^{2}} .
$$

Using the solutions of Ref. 20, we have verified that $S \neq 0$ in the viable regions of the $(\beta, \mu)$-plane (cf. Fig. 1). In these regions, $\tilde{c}_{1} \neq 0, \tilde{c}_{13} \neq 1$, but there exist curves (shown in Fig. 1) on which $\tilde{c}_{13}=0$. If that is the case, one immediately obtains $\omega_{1}(\cos \theta)=0$. In the rest of the viable regions, $\tilde{c}_{13} \neq 0$ and the generic solution to Eq. 20. can be written in terms of hypergeometric functions,

$$
\begin{aligned}
\omega_{1}(\cos \theta)= & \sigma_{12} F_{1}\left(\frac{3-s}{4}, \frac{3+s}{4} ; \frac{1}{2} ; \cos ^{2} \theta\right) \\
& +\sigma_{2} \cos \theta_{2} F_{1}\left(\frac{5-s}{4}, \frac{5+s}{4} ; \frac{3}{2} ; \cos ^{2} \theta\right),
\end{aligned}
$$

with $s \equiv \sqrt{9-8 \tilde{c}_{1}\left(1-\tilde{c}_{13}\right) S / \tilde{c}_{13}}$. This general solution diverges at $\theta=0$ or $\theta=\pi$ unless $\sigma_{1}=\sigma_{2}=0$. In order to prove that $\omega_{n}(\cos \theta)=0$ for any $n$, we can use the recursion theorem and show that if $\omega_{i}(\cos \theta)=0$ for $i<n$, then $\omega_{n}(\cos \theta)=0$. This follows from eq. (15), which at the lowest order in $r-r_{\mathrm{H}}\left(\right.$ using $\omega_{i}(\cos \theta)=0$ for $i<n$ ) gives

$$
\frac{\tilde{c}_{13}\left[-4 \cos \theta \omega_{n}^{\prime}(\cos \theta)+\sin ^{2} \theta \omega_{n}^{\prime \prime}(\cos \theta)\right]}{2 \tilde{c}_{1}\left(1-\tilde{c}_{13}\right)}=n \omega_{n}(\cos \theta) S,
$$

from which we get that $\omega_{n}(\cos \theta)=0$, just like above.

In summary, we have shown that HL gravity does not admit stationary, axisymmetric, slowly rotating BHs. This is alarming because one expects a continuous limit from rotating to nonrotating $\mathrm{BHs}$, as in GR. Also, astrophysical BHs, for which there is nowadays robust evidence [27, do have non-zero spins. More specifically, non-zero measurements for the $g_{t \phi}$ component of the $\mathrm{BH}$ metric ("frame dragging"), which our calculation predicts to be zero in HL gravity, are provided by techniques such as continuum fitting [28] and analyses of the relativistic iron lines [29]. Also, non-zero values of the spin (and thus of the frame dragging) are naturally expected, at least for the "massive" BHs present in galactic centers, based on our current understanding of accretion and mergers during galaxy formation 30. In particular, because the innermost stable circular orbit of HL gravity non-rotating $\mathrm{BHs}$ has a non-zero angular momentum [20, thin-disk accretion around these $\mathrm{BHs}$ would naturally tend to spin them up.

A possible way out is that gravitational collapse never forms BHs in HL gravity. While the BHs of Ref. [20] have been found to form in a perfectly spherical collapse [31, deviations from this idealized picture may give rise to non-trivial rotating configurations of matter and aether. However, for quasi-spherical initial conditions this seems unlikely, unless the higher order term of HL gravity (and the corresponding matter corrections) can somehow halt the collapse. In any case, there is circumstantial evidence that astrophysical $\mathrm{BH}$ candidates possess an event horizon around them [32, which would pose an additional problem for such a scenario. Moreover, rotating "BH mimickers" that do not have an event horizon are typically unstable classically due to the so-called ergoregion instability [33.

Note that in Refs. [20, 21] another set of static, spherically symmetric, asymptotically flat solutions with a metric horizon was found. These solutions were discarded because they exhibit a finite area singularity on the spin- 0 horizon (the causal boundary of the spin- 0 mode, which can be inside the metric horizon). It is indeed hard to imagine how such spacetimes may form from collapse, but one might conceivably replace part of the interior with a configuration of matter and aether, so as to "cover" the singularity (cf. the discussion about the universal horizon instability in Ref. 22]). These configurations may have a metric horizon surrounding the matteraether configuration, and might replace the spherical BHs used as seeds for the slowly rotating solutions studied in this Letter.

Finally, stationary, axisymmetric BHs might possibly exist, for which the aether does not share these symmetries (but its stress-energy tensor does). This may be similar to the "stealth" solutions of $2+1$ gravity with a nonminimally coupled and self-interacting scalar field 34].

Acknowledgments: We thank Ted Jacobson, Luis 
Lehner, Paolo Pani and Sergey Sibiryakov for many enlightening discussions and insightful comments of an earlier version of this manuscript. EB acknowledges support from a CITA National Fellowship at the University of Guelph. TPS acknowledges partial financial support provided under a Marie Curie Career Integration Grant and the "Young SISSA Scientists Research Project" scheme 2011-2012.

Note added: A subtlety in the dynamical equivalence between Hořava-Lifshitz gravity and Einstein-aether theory has been missed and this has seriously affected the conclusions of this paper. Please see arXiv:1212.1334 for a full discussion.

[1] T. Jacobson and D. Mattingly, Phys. Rev. D 64, 024028 (2001)

[2] P. Hořava, Phys. Rev. D 79, 084008 (2009)

[3] M. Visser, Phys. Rev. D 80 (2009) 025011

[4] M. Visser, arXiv:0912.4757 [hep-th]

[5] T. P. Sotiriou, J. Phys. Conf. Ser. 283, 012034 (2011)

[6] D. Blas, O. Pujolas and S. Sibiryakov, Phys. Rev. Lett. 104, 181302 (2010)

[7] T. P. Sotiriou, M. Visser and S. Weinfurtner, Phys. Rev. Lett. 102, 251601 (2009)

[8] T. P. Sotiriou, M. Visser and S. Weinfurtner, JHEP 0910, 033 (2009)

[9] P. Horava and C. M. Melby-Thompson, Phys. Rev. D 82, 064027 (2010)

[10] D. Vernieri and T. P. Sotiriou, Phys. Rev. D 85, 064003 (2012)

[11] C. Charmousis, et al., JHEP 0908, 070 (2009)

[12] D. Blas, O. Pujolas and S. Sibiryakov, JHEP 0910, 029 (2009)

[13] A. Papazoglou and T. P. Sotiriou, Phys. Lett. B 685, 197 (2010)

[14] I. Kimpton and A. Padilla, JHEP 1007, 014 (2010)
[15] D. Blas, O. Pujolas and S. Sibiryakov, JHEP 1104, 018 (2011)

[16] D. Blas, O. Pujolas and S. Sibiryakov, Phys. Lett. B 688, 350 (2010)

[17] S. Liberati, L. Maccione and T. P. Sotiriou, arXiv:1207.0670 [gr-qc]

[18] T. Jacobson, Phys. Rev. D 81, 101502 (2010) [Erratumibid. D 82, 129901 (2010)]

[19] T. P. Sotiriou, M. Visser and S. Weinfurtner, Phys. Rev. D 83, 124021 (2011)

[20] E. Barausse, T. Jacobson and T. P. Sotiriou, Phys. Rev. D 83, 124043 (2011)

[21] C. Eling and T. Jacobson, Class. Quant. Grav. 23, 5643 (2006) [Erratum-ibid. 27, 049802 (2010)]

[22] D. Blas and S. Sibiryakov, Phys. Rev. D 84, 124043 (2011)

[23] J. B. Hartle, Astrophys. J. 150 (1967) 1005; J. B. Hartle and K. S. Thorne, Astrophys. J. 153, 807 (1968)

[24] B. Z. Foster, Phys. Rev. D 72, 044017 (2005)

[25] J. W. Elliott, G. D. Moore and H. Stoica, JHEP 0508, 066 (2005).

[26] P. T. Chrusciel, J. L. Costa and M. Heusler, Living Rev. Rel. 15, 7 (2012).

[27] R. Narayan, New J. Phys. 7, 199 (2005)

[28] J. E. McClintock, et al. Class. Quant. Grav. 28, 114009 (2011)

[29] A. C. Fabian, et al., Mon. Not. Roy. Astron. Soc. 238, 729 (1989); L. W. Brenneman, et al., Astrophys. J. 736, 103 (2011)

[30] E. Berti and M. Volonteri, Astrophys. J. 684, 822 (2008); N. Fanidakis, et al., Mon. Not. Roy. Astron. Soc. 410, 53 (2011); E. Barausse, Mon. Not. Roy. Astron. Soc. 423, $2533(2012)$

[31] D. Garfinkle, C. Eling and T. Jacobson, Phys. Rev. D 76, 024003 (2007)

[32] A. E. Broderick and R. Narayan, Astrophys. J. Lett. 638, L21 (2006); A. E. Broderick, A. Loeb and R. Narayan, Astrophys. J. 701, 1357 (2009)

[33] P. Pani, et al., Phys. Rev. D 82, 044009 (2010)

[34] E. Ayon-Beato, C. Martinez and J. Zanelli, Gen. Rel. Grav. 38, 145 (2006) 